frequency of malignancies in BS represents that in the general population, this is the first report of a paediatric patient developing AML following treatment for ALL. This girl was found to carry two novel $B L M$ mutations, c.1221-1G $>A$ and c.1624delG. This case documents the short interval at which treatment-related myeloid malignancy may occur in a child with BS and implies a fundamental role for BLM for normal haematopoiesis, in particular in the presence of genotoxic stress. It demonstrates the importance of molecular analysis in atypical cases of childhood malignancies. Novel approaches are required to improve treatment for these individuals as optimal dose delivery to often aggressive malignancies is hindered by extreme sensitivity to treatment toxicity.

\section{G175 THE IMPACT OF SOCIAL ISSUES ON THE HEALTH OF CHILDREN PRESENTING TO TWO TERTIARY PAEDIATRIC SICKLE CELL CENTRES}

doi:10.1136/archdischild-2013-304107.187

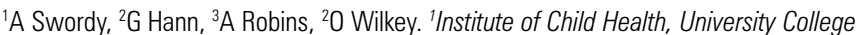
London, London, UK; ' ${ }^{2}$ epartment of Paediatrics, North Middlesex University Hospital, London, UK; ${ }^{3}$ Department of Paediatrics, Whittington Hospital, London, UK

Background Sickle cell disease (SCD) is an inherited blood disorder which affects 1 in every 2000 children born in the UK. Specialist clinics for children with SCD have identified that attending children live in areas of high deprivation, with many experiencing child protection issues.

Aims This study aims to examine the burden of social issues on this population of children with SCD, and investigate the impact of these factors on adherence to medication, clinic attendance, and inpatient admissions.

Method A retrospective notes audit of 360 children from two tertiary sickle cell referral centres was carried out and information on patient and family characteristics, social background and child protection issues, compliance with penicillin and immunisations was collated in line with national guidelines.

Results Housing problems were present in $25.8 \%$ of patients, immigration in $10 \%$ and child protection issues in $9.2 \% .22 .7 \%$ of patients were from single-parent families. The hospitals had good records for immunisations, (93.3\% up-to-date), but poor clinic attendance, (mean attendance rate $71.87 \%$ ). Attendance was higher with a more severe phenotype but not significantly different with social issues. Poor compliance was identified in $15 \%$ of patients. Strong associations exist between poor compliance and poor clinic attendance, not being fully immunised and the presence of parental SCD. There was a significantly higher incidence of inpatient admissions in patients with housing issues as compared to those without at both hospitals (Unit 1: $p \leq 0.017$; Unit $2 \mathrm{p} \leq 0.027$; combined in multivariate model $\mathrm{p} \leq 0.04)$. There was also a higher incidence of inpatient admissions in patients with immigration issues than those without, however this was not statistically significant $(\mathrm{p} \leq 0.28)$. There was no difference in inpatient admissions between single-parent and two-parent households.

Conclusion The significant burden of social problems and child protection issues in the paediatric sickle cell population studied must be recognised due to the impact on patients' health, admissions to hospital, ability to attend clinic, and maintain compliance. Further research on causation of poor clinic attendance and poor compliance would reduce waste and improve health service efficiency, as well as being of considerable benefit to patient health and wellbeing.

\section{G176 THE NATIONAL CANCER SURVIVORSHIP INITIATIVE: A NATIONAL APPROACH TO IMPROVE THE QUALITY OF AFTERCARE FOR SURVIVORS OF CHILDHOOD CANCER}

doi:10.1136/archdischild-2013-304107.188
${ }^{1} \mathrm{H}$ Jenkinson, ${ }^{2,8} \mathrm{~F}$ Gibson, ${ }^{3 \mathrm{P}}$ Morris,, $4.9 \mathrm{M}$ Stevens, ${ }^{5} \mathrm{H}$ Hatcher, ${ }^{6} \mathrm{~A}$ Glaser, ${ }^{7} \mathrm{~A}$ Toogood, ${ }^{2} \mathrm{G}$ Levitt. 'Department of Paediatric Oncology, Birmingham Children's Hospital NHS Foundation Trust, Birmingham, UK; ${ }^{2}$ Department of Paediatric Oncology, Great Ormond Street Hospital for Children NHS Foundation Trust, London, UK; ${ }^{3}$ NHS Improvement - Cancer, Institute of Health Service Management, London, UK; ${ }^{4}$ Department of Paediatric Oncology, Bristol Royal Hospital for Children, Bristol, UK; ${ }^{5}$ Department of Oncology, Addenbrooke's Hospital, Cambridge, UK; ${ }^{6}$ Department of Paediatric Oncology, Leeds Teaching Hospitals NHS Trust, Leeds, UK; 'Department of Endocrinology, University Hospitals Birmingham NHS Foundation Trust, Birmingham, UK; ${ }^{8}$ London South Bank University, London, UK; ${ }^{9}$ University of Bristol, Bristol, UK

Aims The National Cancer Survivorship Initiative [NCSI] (a partnership between the Department of Health, NHS Improvement, third sector organisations and user representatives) was established to improve the care provided for patients 'living with and beyond cancer'. It sought to work with cancer survivors to inform new models of care. The focus was to shift from traditional hospitalbased models of aftercare to informed personalised patient care. The NCSI children and young people's [CYP] work stream sought specifically to design functional, cost-effective models of care underpinned by evidence.

Methods A multi-professional, multi-staged approach was used Following an initial period of consultation, centres around England were invited to submit a service improvement project. Ten sites providing cancer care for children and young people were selected. Each site tested potential areas of change across newly-defined patient pathways, each led by an experienced clinician with service improvement input as well as significant input from young people and parents. The aim was to gather baseline evidence, test concepts and disseminate reports nationally to share learning more widely. Four centres were invited to test the models of care.

Results Evidence emerged in support of: clinical risk stratification to allow patients to receive care tailored to their individual need, personalised patient information in the form of a treatment summary and care plan, holistic needs assessment, alternative models of care to replace traditional consultant-led follow up including nurseled care, telephone support and supported self management, psychosocial screening. Emerging from this work three different CYP patient pathways have been developed which are felt to represent gold standard care for survivors of childhood cancer at different stages of follow up and of differing risk groups.

Conclusions Comprehensive stakeholder collaboration, robust service improvement strategies and sharing of good practise and learning, have resulted in the development of evidence-based and sustainable packages of care for the growing cohort of survivors of childhood and young adult cancer. Spread and implementation are underway, using face-to-face and web-based interactive formats, to influence service delivery that can be adapted to suit local circumstances and resources.

\section{G177 A SINGLE-CENTRE EXPERIENCE OF CENTRAL VENOUS LINES IN PAEDIATRIC HAEMATOLOGY/ONCOLOGY PATIENTS OVER FIVE YEARS}

doi:10.1136/archdischild-2013-304107.189

${ }^{1}$ RYM Toh, ${ }^{1} \mathrm{H}$ Mackay, ${ }^{2} \mathrm{~A}$ Isaac, ${ }^{3} \mathrm{C}$ Keys, ${ }^{2} \mathrm{AB}$ Edgar, ${ }^{1,2} \mathrm{WH}$ Wallace. ${ }^{1} \mathrm{C}$ ollege of Medicine and Veterinary Medicine, University of Edinburgh, Edinburgh, UK; ${ }^{2}$ Department of Paediatric Haematology and Oncology, Royal Hospital for Sick Children, Edinburgh, UK ${ }^{3}$ Department of Surgical Paediatrics, Royal Hospital for Sick Children, Edinburgh, UK

Background and Aims Safe central venous access is required for the management of paediatric patients with solid and haematological malignancies. The objective of this study was to retrospectively review central venous lines (CVL) experience in a single centre over a five-year period between 2007 and 2012 to determine the prevalence of line-associated complications and predisposing factors for premature line removal. 